Off 34 tranexamic acid errors (from 13 countries), 7 occurred in India. 8 European union countries reported 15 (out of 28) potassium chloride errors. Neuromuscular blocking drugs related events were widespread (21 errors from 13 countries). Cardiovascular drug incidents (34) were mainly reported from the USA (9) and UK (6).

Table 2 summarises some issues related to pharmaceutical industry and clinical practice.

Recurrent human factors identified are summarised in table 3 .

Conclusions Manufacturing of look-alike of LA, fentanyl, normal saline and several high-risk ampoules (e.g. tranexamic acid, $\mathrm{KCl}$, digoxin, NMBDs) or vials is one major uniform factor. Robust organisational, supervisory and local clinical practices are needed to correct latent human failures.Universal applications of four recommendations ${ }^{1-4}$ (table 3) would prevent drug administration errors during neuraxial anaesthesia or analgesia.

\section{EFFICACY AND SAFETY OF INTRATHECAL MORPHINE FOR ANALGESIA AFTER LOWER JOINT ARTHROPLASTY: A SYSTEMATIC REVIEW AND META-ANALYSIS WITH META-REGRESSION AND TRIAL SEQUENTIAL ANALYSIS}

${ }^{1} \mathrm{E}$ Gonvers*, ${ }^{2} \mathrm{~K}$ El-Boghdadly, ${ }^{3} \mathrm{~S}$ Grape, ${ }^{1} \mathrm{E}$ Albrecht. 'Lausanne University Hospital, Lausanne, Switzerland; ' ${ }^{2}$ Guy's and St Thomas' NHS Foundation Trust, London, UK; ${ }^{3}$ Hôpital du Valais, Sion, Switzerland

\subsection{6/rapm-2021-ESRA.39}

Background and Aims Widespread adoption of intrathecal morphine into clinical practice is hampered by concerns of its potential side-effects. We undertook a systematic review, metaanalysis and trial sequential analysis with the primary objective of determining the efficacy and safety of intrathecal morphine. Our secondary objective was to determine the dose associated with greatest efficacy and safety.

Methods We systematically searched the literature for any trials comparing intrathecal morphine with a control group in patients undergoing hip, knee arthroplasty under spinal anaesthesia. Our primary efficacy outcome was rest pain score (010) at $8-12 \mathrm{~h}$; our primary safety outcome was the rate of PONV within $24 \mathrm{~h}$.

Results Twenty-nine trials including 1814 patients were identified. Rest pain score at $8-12 \mathrm{~h}$ was significantly reduced in the intrathecal morphine group with a mean difference $(95 \% \mathrm{CI})$ of $-1.7(-2.0,-1.3), \mathrm{I}^{2}=71 \%, \mathrm{p}<0.0001$, without subgroup difference between doses $(\mathrm{p}=0.35)$. Intrathecal morphine increased postoperative nausea and vomiting with a risk ratio $(95 \% \mathrm{CI})$ of $1.4(1.2,1.6), \mathrm{I}^{2}=4 \%$, $\mathrm{p}<0.0001$. However, a subgroup analysis according to doses revealed that rates of PONV within $24 \mathrm{~h}$ was similar between groups with doses of $100 \mu \mathrm{g}$, while the risk significantly increased with doses above ( $p$ value for subgroup difference $=0.03$ ). The quality of evidence for our two primary outcomes was high and moderate-to-high for the secondary outcomes.

Conclusions There is high level evidence that intrathecal morphine provides effective analgesia after lower limb arthroplasty but at the expense of an increased profile of sideeffects. However, a dose of $100 \mu \mathrm{g}$ represents a ceiling dose for analgesia and a threshold dose for increased rate of PONV.

\section{DEVELOPMENT OF A REGIONAL ANAESTHESIA SERVICE FOR RIB FRACTURES: A QUALITY IMPROVEMENT PROJECT}

A Ee* ${ }^{*}$ T Rees. Royal Gwent Hospital, Newport, UK

\subsection{6/rapm-2021-ESRA.40}

Background and Aims Trauma audit research network (TARN) data for 2018 indicated that 100 patients are admitted with chest wall injuries. Retrospective audit of referrals confirmed long length of stay (median 14 days), with 59\% requiring level $2 / 3$ care and $11 \%$ mortality risk.

Regional anaesthesia was offered to less than $25 \%$ of patients despite $63 \%$ reporting severe pain. We decided to introduce an Erector Spinae plane (ESP) catheter service for these patients with aims of reducing length of stay and pain scores.

Methods We set up an email alert system, where TARN data collectors notified us when patients were admitted through the Emergency department with rib fractures. Using Whatsapp, we organised a group of regional anaesthetists who were willing to provide an ESP service.

Results Mean length of hospital stay was reduced from 10 to 7 days after introduction of the service with significant reduction in variability.

Mean pain scores improved from $8.9 / 10$ to $5 / 10$ with an average improvement of pain score of 2.78 points on a numerical scale out of $10 .(n=9)$

Conclusions This was an easy, cost-neutral intervention that has made a noticeable difference in a short time frame. The small reduction in pain scores may be explained by the presence of concurrent injuries. Ongoing improvements in training should improve reliability of catheter placement and reduce practitioner variation.
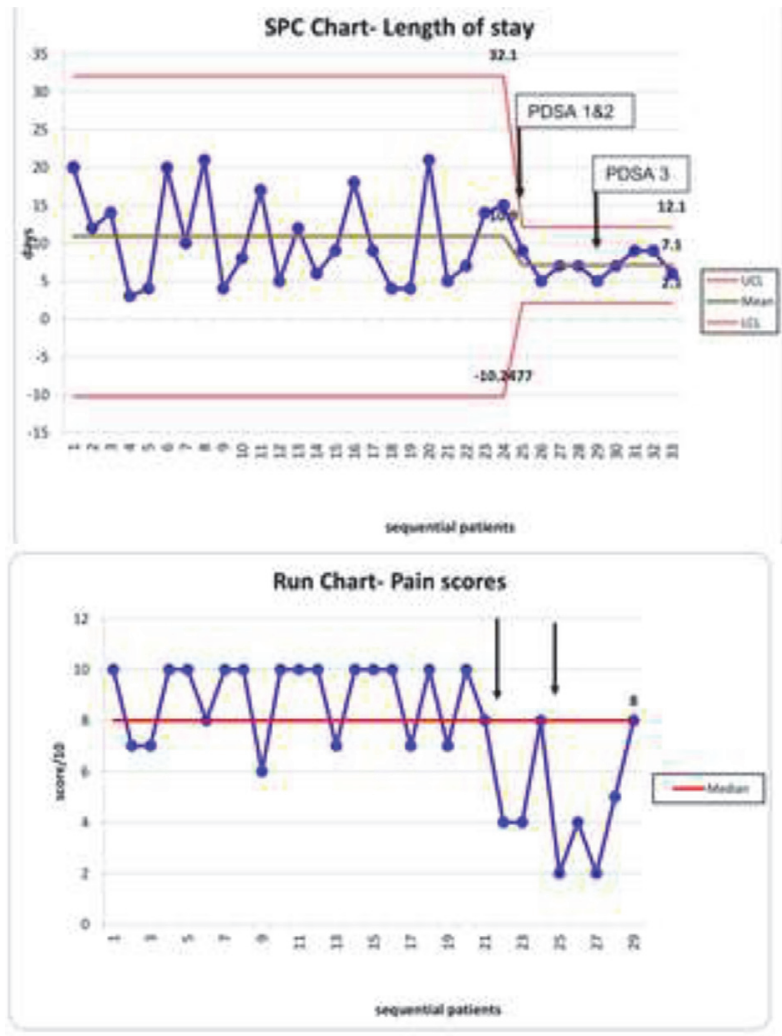

Abstract 40 Figure 1 